\title{
Uso referido de medicamentos e suplementos alimentares nos atletas selecionados para controle de doping nos J ogos Sul-Americanos
}

\author{
Eduardo Henrique De Rose ${ }^{1}$, Marta Goldman Feder², Paulo Rodrigo Pedroso ${ }^{3}$ e André Zanette Guimarães ${ }^{4}$
}

\section{RESUMO}

O objetivo do presente estudo foi descrever a prevalência do uso de medicamentos e suplementos alimentares em atletas que participaram dos controles obrigatórios de dopagem dos VII J ogos Desportivos Sul-Americanos, realizados em quatro cidades brasileiras, analisando estatisticamente um quesito proposto no controle de dopagem e relativo às substâncias utilizadas nos dias que antecederam a competição. Foram analisados dados coletados em 234 atletas de 25 esportes dos J ogos, 136 do sexo masculino e 98 do feminino, distribuídos entre os 13 países participantes. Constatou-se que no controle de doping, $44 \%$ dos atletas informaram a utilização de medicamentos até três dias antes da competição. Estes medicamentos foram classificados em antiinflamatórios não esteróides (AINEs) (24,8\%), analgésicos (15,9\%), antibióticos $(4,3 \%)$, antigripais (3\%) e outros medicamentos (19,3\%). Relataram o uso de suplementos alimentares $50 \%$ de atletas da amostra estudada, sendo este grupo dividido em vitaminas $(39,7 \%)$, sais minerais $(21,9 \%)$, aminoácidos $(18,9 \%)$ e outras substâncias $(13,3 \%)$. Os autores concluem que há um uso exagerado de antiinflamatórios e analgésicos em várias modalidades desportivas, o que causa preocupação em termos de controle de sintomatologia dos atletas em competição, além de uso excessivo de suplementos alimentares, para os quais não existe indicação específica, e que podem ocasionar um eventual resultado analítico adverso no controle de doping por contaminação ou manipulação.

\section{ABSTRACT \\ Referred use of medication and dietary supplements in athletes selected for doping control in the South-American Games}

The objective of the present study was to describe the prevalence of medicine and dietary supplements use in athlete who participated in the mandatory doping controls of the VII South American Sports Games, which took place in four Brazilian cities. One issue considering the doping control and relative to substances used in the days that preceded the competition was statistically analyzed. The authors analyzed data collected from 234 athletes of 25 sports participating in the Games, being these athletes 136 males and 98 females, distributed among the 13 participating coun-

1. Professor Titular. Disciplina de Medicina do Esporte. Universidade do Rio Grande do Sul, Porto Alegre, RS.

2. Pesquisador. Serviço de Traumatologia. Hospital de Clínicas de Porto Alegre. Universidade do Rio Grande do Sul, Porto Alegre, RS.

3. Mestre em Endocrinologia. Serviço de Endocrinologia. Hospital de Clínicas de Porto Alegre. Universidade do Rio Grande do Sul, RS.

4. Doutorando da Faculdade de Medicina. Universidade Luterana do Brasil, Canoas, RS.

Recebido em 7/5/06. Versão final recebida em 3/8/06. Aceito em 24/8/06.

Endereço para correspondência: Eduardo Henrique De Rose, Rua Felipe Becker, 95 - Bairro Três Figueiras - 91330-250 - Porto Alegre, RS. Email: ehderose@terra.com.br

\author{
Palavras-chave: J ogos. Atletas. Uso de medicação. Suplementos. Doping \\ Keywords: Games. Athletes. Use of medication. Dietary supplements. Dop- \\ ing. \\ Palabras-clave: J uegos. Atletas. Uso de medicación. Suplementos. Doping.
}

tries. According to the questionnaire conducted in the doping control, 44\% athletes informed some substances used up to three days before the competition. The medications were classified into non-steroidal anti inflammatory (NSAI) (24.8\%), analgesics (15.9\%), antibiotics (4.3\%), cold medicine (3\%) and other medicines (19.3\%). Moreover, 50\% of athletes reported the use of dietetic supplements, being this group divided into vitamins (39.7\%), minerals $(21.9 \%)$, amino acids (18.9\%) and other substances (13.3\%). The authors concluded that there was an overuse of antiinflammatory and analgesic medication in many sports modalities, which raises a concern in terms of control of symptoms of the athletes in competitions. Besides that, there was an important use of dietary supplements without specific indication. Such fact may cause an eventual adverse analytical finding in the control of doping by contamination or manipulation.

\section{RESUMEN}

Uso referido de medicinas y suplementos alimenticios en los atletas seleccionados para control de doping en los J uegos Sudamericanos

El objetivo del presente estudio ha sido el de describir la prevalencia del uso de medicamentos y suplementos alimenticios en atletas que participaron de los controles obligatorios de dopaje de Ios VII J uegos Deportivos Sudamericanos, realizados en cuatro ciudades brasileñas, analizando estadísticamente un requisito propuesto en el control de dopaje relativo a substancias utilizadas durante días que antecedieron a la competición. Fueron analizados datos colectados en 234 atletas de 25 deportes participantes de los J uegos, de los cuales 136 participantes eran del sexo masculino y 98 participantes del sexo femenino, distribuidos entre los 13 países participantes. Se constató que en el control de doping $44 \%$ de los atletas informaron la utilización de medicinas de hasta tres días antes de la competición. Estas medicinas fueron clasificadas en antiinflamatorios no esteróides (AINES) (24,8\%), analgésicos (15,9\%), antibióticos (4,3\%), antigripales (3\%) y otros medicamentos $(19,3 \%)$. También relataron el uso de suplementos alimenticios $50 \%$ de los atletas de la muestra estudiada, y este grupo fue dividido en vitaminas (39.7\%), sales minerales (21,9\%), aminoácidos $(18,9 \%)$ y otras substancias $(13,3 \%)$. Los autores concluyen que hay un uso exagerado de antiinflamatorios y analgésicos en varias modalidades deportivas, lo que causa preocupación en téminos de control de sintomatología de los atletas en competición, y ocurre en competición un uso excesivo de suplementos alimenticios, para los cuales no existe indicación específi$c a, y$ que pueden ocasionar un eventual resultado analítico adverso en el control de doping por contaminación o manipulación. 


\section{INTRODUÇÃO}

Os J ogos Desportivos Sul-Americanos foram iniciados no ano de 1978, na cidade de La Paz, Bolívia, e a sua sétima edição foi realizada no Brasil, nas cidades de Curitiba, São Paulo, Rio de J aneiro e Belém do Pará. Neste evento, foi realizado pela Comissão Médica da Organização Desportiva Sul-Americana (ODESUR) o controle antidoping de atletas, conforme exigência do Código Internacional Antidoping da Agência M undial Antidoping (WADA)(1), selecionados randomicamente de forma dirigida entre os atletas participantes. Um dos quesitos respondidos pelos atletas durante este procedimento, de acordo com os padrões do Comitê Olímpico Brasileiro $(\mathrm{COB})^{(2)}$, foi o de medicamentos usados nos últimos três dias. A análise deste quesito propiciou a elaboração do presente estudo.

As informações constantes do Formulário de Controle de Dopagem foram analisadas quanto a medicamentos e suplementos alimentares, para avaliar o perfil de utilização dos principais atletas da América do Sul, presentes nesta competição. O conhecimento desta realidade permite dar uma idéia de que necessidades os atletas eventualmente possuem ou quais benefícios visam obter.

A literatura nacional menciona apenas alguns poucos estudos desta natureza. Barros Neto(3) chama a atenção para o excessivo uso de agentes ergogênicos farmacológicos, completamente em desacordo com as necessidades nutricionais dos atletas e as evidências publicadas na literatura. Em diretriz da Sociedade Brasileira de Medicina do Esporte sobre este tema, Carvalho et al.(4) descrevem o uso excessivo de suplementos alimentícios em nosso país, sem prescrição médica e com riscos para a saúde.

$\mathrm{Na}$ área intemacional, distintos autores constataram o alto uso de suplementos por atletas, desaconselhando este procedimento(5-7). Analisando a utilização deste tipo de produto em atletas de alto nível, Burke ${ }^{(8)}$ chama a atenção para a possibilidade de surgirem resultados analíticos adversos no controle de doping, em razão da ausência de controle de qualidade e deficiências eventuais nos rótulos destes produtos, mencionando ainda que isto se dá exclusivamente por erro dos atletas, e neste caso os dirigentes esportivos não devem escusá-los por isso. Especificamente em eventos internacionais desportivos e como conseqüência de recomendação da WADA(9), estudos foram realizados nos atletas controlados nos J ogos Olímpicos de Sidney e posteriormente publicados por Corrigan e Kazlauskas ${ }^{(10)}$. Em função do percentual elevado de suplementos usados pelos atletas nesta Olimpíada, uma pesquisa foi encomendada pelo Comitê Olímpico Internacional (COI) e realizada com 634 suplementos alimentares de 13 países diferentes no Instituto de Bioquímica de Colônia, por Geyer et al.(11), usando a mesma técnica proposta anteriormente por estes autores ${ }^{(12)}$. Seu objetivo era verificar a presença ou não de próhormônios (principalmente testosterona e nandrolona) que pudessem causar resultados analíticos adversos no controle antidoping. Deve-se mencionar que um estudo anterior ao de Sydney, ainda que não publicado, já havia sido feito em nosso continente por D'Angelo(13) nos J ogos Pan-Americanos de Mar del Plata, Argentina, em 1995.

O uso de suplementos, conforme evidenciado em referência anterior(9), pode causar um resultado analítico adverso no controle de dopagem em função de contaminações casuais destes produtos, uma vez que as empresas produtoras não são obrigadas a declarar integralmente sua composição, além de não seguirem em sua maioria as normas técnicas ideais para produção de substâncias em laboratório, conhecidas internacionalmente como Good Manufactoring Practices (GMP).

\section{OBJ ETIVO GERAL}

Descrever a prevalência do uso de medicamentos e suplementos alimentares em atletas que participaram dos controles obriga- tórios de dopagem dos VII J ogos Sul-Americanos de 2002, realizados em quatro cidades brasileiras, analisando a resposta ao quesito medicamentos utilizados nos últimos três dias.

\section{MÉTODO}

Foram analisadas as informações coletadas em 242 controles de doping, feitos em 234 atletas de 25 esportes com um total de 33 modalidades participantes dos VII J ogos Desportivos Sul-Americanos. Estes atletas dividiram-se em 136 do sexo masculino e 98 do feminino, distribuídos entre os 13 países participantes, sendo 11 da América do Sul (Argentina, Bolívia, Brasil, Chile, Equador, Guiana, Paraguai, Peru, Suriname, Uruguai e Venezuela), um da América Central (Panamá) e um do Caribe Holandês (Aruba).

A amostra foi constituída de atletas selecionados entre os participantes, sendo formada por medalhistas de ouro, prata e bronze vencedores de provas individuais ou por equipe. Também participaram esportistas que foram escolhidos aleatoriamente, por sorteio ou designação por parte do delegado técnico da competição.

A coleta de dados foi realizada por um grupo de especialistas brasileiros em controle de dopagem, treinado dentro da padronização internacional proposta pela WADA, sendo que os Formulários de Controle de Doping foram preenchidos com informações solicitadas aos atletas selecionados. Este formulário é composto por três vias: a primeira vai para o Comitê Organizador dos J ogos, a segunda, sem identificar o atleta, segue para o Laboratório de Controle de Doping, e a terceira é entregue ao desportista.

O banco de dados deste estudo foi construído através da digitação da resposta ao quesito medicações anteriores, na via original do formulário, e foi elaborada uma planilha eletrônica que dividiu os atletas em modalidades, sexo, país, medicamentos e suplementos alimentares.

Os medicamentos foram divididos nas cinco seguintes categorias: antiinflamatórios não esteróides (AINEs), analgésicos, antibióticos, antigripais e outros. Neste último item incluiu-se toda a gama de medicamentos que tiveram apenas uma citação eventual, como anticoncepcionais orais e laxantes. Para a classificação correta de medicamentos da farmacopéia dos demais países participantes, foram consultados especialistas em Medicina do Esporte destas localidades.

Os suplementos alimentares foram divididos nas quatro seguintes categorias: vitaminas, minerais, aminoácidos e outros. Nesta última classe foram considerados basicamente isotônicos e hidratos de carbono. Nas duas divisões, consideraram-se as substâncias encontradas com maior freqüência nos estudos anteriores de Corrigan e Kazlauskas( ${ }^{(10)}$ e D'Angelo ${ }^{(13)}$.

Este banco de dados foi submetido à análise estatística pelo SPSS em sua versão 11 e o Microsoft Excel. Os resultados foram demonstrados através de estatística descritiva em freqüências e percentuais.

\section{RESULTADOS}

A distribuição dos atletas que compõem esta amostra se apresenta da seguinte maneira: $63,63 \%$ por medalhistas de ouro, $23,13 \%$ por medalhistas de prata e bronze e $13,24 \%$ escolhidos aleatoriamente. Esta distribuição está representada no gráfico 1.

Constatou-se que dos 234 atletas de vários países, 157 (66,25\%) usaram medicamentos imediatamente antes ou no momento da competição. Estes medicamentos foram classificados da seguinte forma: 58 (36,9\%), AINEs; 37 (23,6\%), analgésicos; 10 (6,4\%), antibióticos; $7(4,5 \%)$, antigripais; e $45(28,6 \%)$, outros medicamentos. O gráfico 2 mostra a distribuição percentual no grupo de medicamentos proposta pelos autores.

Relataram uso de suplementos alimentares 117 atletas (50\%) da amostra estudada. Neste grupo consumiram vitaminas 93 
$(42,47 \%)$, sais minerais $51(23,28 \%)$, aminoácidos $44(20,09 \%)$ e outras substâncias 31 (14,15\%). O gráfico 3 mostra a distribuição percentual no grupo de suplementos proposto pelos autores.

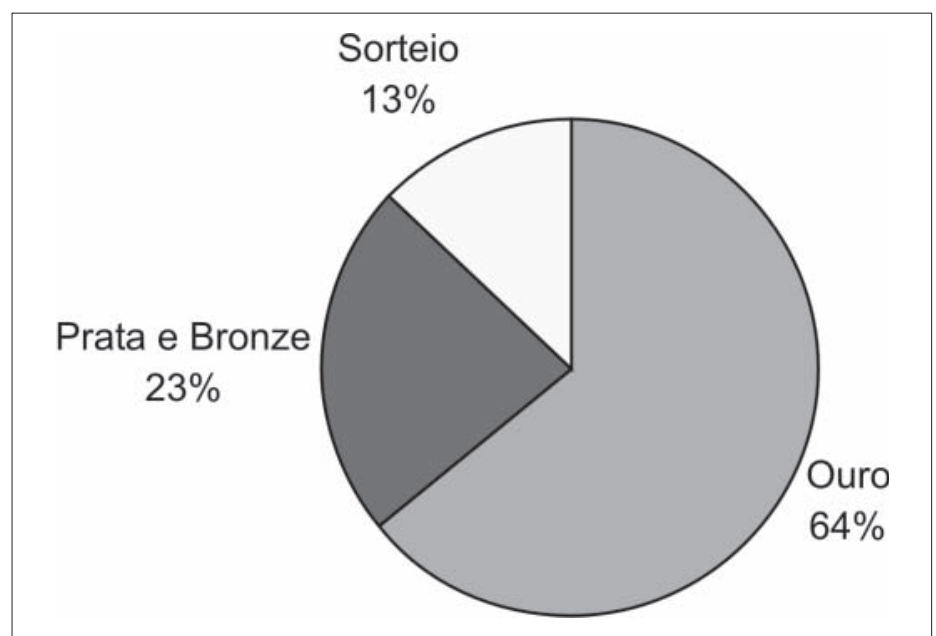

Gráfico 1 - Distribuição dos controles de doping entre os atletas participantes

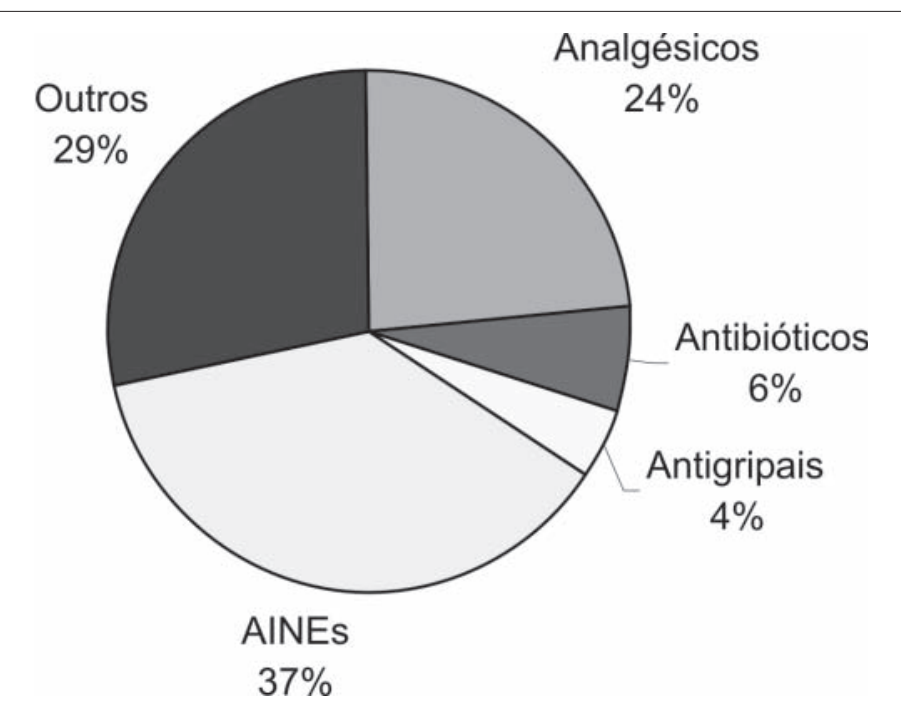

Gráfico 2 - Distribuição percentual para medicamentos na amostra

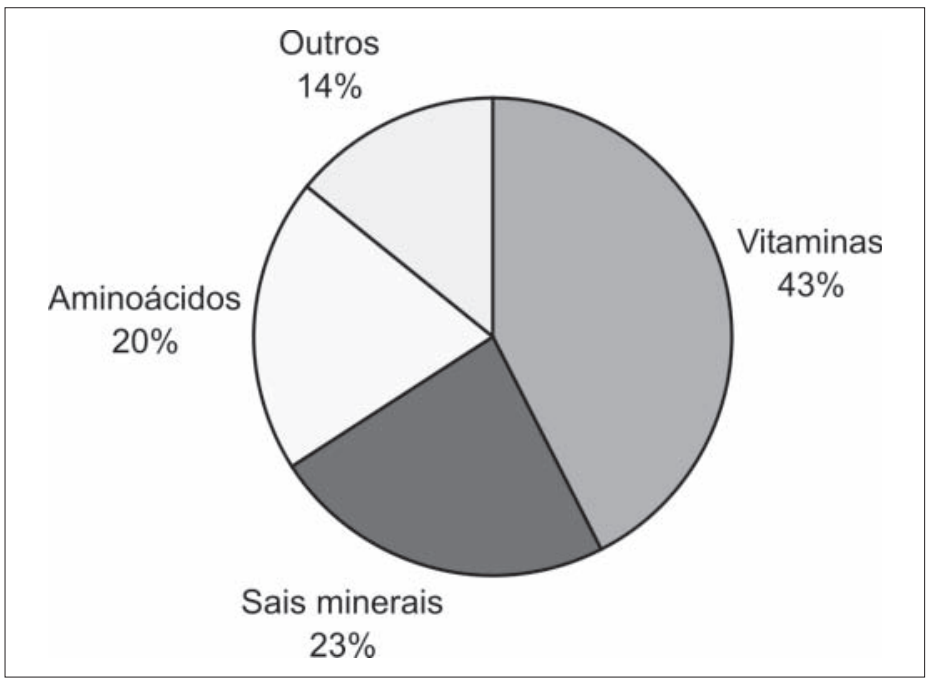

Gráfico 3 - Distribuição percentual para suplementos na amostra
Em termos de uso de medicamentos ou de suplementos alimentares, pode existir uma múltipla utilização por atleta, razão pela qual os números podem ser, evidentemente, maiores do que a população estudada. Em relação ao gênero e consumo geral, verifica-se que os homens usam mais medicamentos e suplementos alimentares que as mulheres, sem diferença significativa no que se refere à distribuição por países.

\section{DISCUSSÃO}

Os autores optaram pela classificação nas categorias, conforme descrito em Método, por entenderem que, em cada um dos grupos (medicamentos e suplementos), as subdivisões refletem a realidade de consumo pelo atleta.

Os medicamentos mais usados foram os da classe de AINEs, expressando uma tendência percebida na clínica médica, que é a necessidade do tratamento de lesões advindas do treinamento para a competição. A classe de analgésicos foi a segunda mais usada e corrobora a explicação acima.

A preocupação reside no fato de que, se há real necessidade do uso destas medicações, quer por prescrição médica, quer por automedicação, deve existir concomitantemente uma condição de lesão/sofrimento para o atleta quando da competição. Há que se questionar se isto ocorre por excesso de treinamento, pela forma como o treinamento é feito, por situações de fragilidade fisiológica ou por outras variáveis que poderiam ser alteradas, determinando maior uso destas medicações. É importante lembrar que a amostra é constituída principalmente por atletas vencedores em suas competições, o que pressupõe um elevado grau de desempenho.

Os suplementos alimentares mais usados foram as vitaminas, seguidas por sais minerais, também expressando uma cultura de alto consumo, uma vez que não existe na literatura específica qualquer indicação para o uso dessas substâncias.

Os autores acreditam que o uso indiscriminado de suplementos, também evidenciado em estudos anteriores, possa vir a causar um aumento de resultados analíticos adversos no controle de doping, por desconhecimento do atleta quanto ao produto que está sendo consumido. Sem ter clara a procedência do mesmo, não há como prevenir-se de manipulações intencionais ou contaminações industriais no processamento destas substâncias, já que as empresas fabricam vários tipos de produtos sem padronização das normas técnicas ideais.

Existe a possibilidade de que, na coleta de dados, o atleta se abstenha de informar as medicações efetivamente usadas ao especialista em controle de doping. Em função deste fato, a utilização de fármacos e suplementos pode eventualmente ser maior do que a referida, o que certamente consideramos como uma das limitações do presente artigo, pois os atletas não são evidentemente obrigados a informar toda a verdade no momento do controle.

\section{CONCLUSÃO}

Os autores concluem que há um uso exagerado de antiinflamatórios e analgésicos em várias modalidades desportivas, distribuído nos diversos países da América do Sul, em virtude da preocupação em termos de controle de sintomatologia dos atletas em competição. O uso excessivo de suplementos alimentares não apresenta indicação na literatura e pode ocasionar um eventual resultado analítico adverso no controle de doping.

Os resultados refletem padrão de comportamento similar ao verificado em outros estudos realizados por vários autores em competições intemacionais onde participam atletas de alto nível.

Todos os autores declararam não haver qualquer potencial conflito de interesses referente a este artigo. 


\section{REFERÊNCIAS}

1. WADA-AMA. World Anti-Doping Code (Version 3). Montreal: WADA, 2003.

2. De Rose EH, Feder MG, Bento RM, Aquino Neto FR. Informações sobre o uso de medicamentos no esporte. 4a ed. Rio de J aneiro: COB, 2004.

3. Barros Neto TL. A controvérsia dos agentes ergogênicos: estamos subestimando os efeitos naturais da atividade física? Arq Bras Endocrinol Metab. 2001; 45(2):121-2.

4. Carvalho T, Rodrigues T, Meyer F, Lancha J r AH, De Rose EH. Modificações dietéticas, reposição hídrica, suplementos alimentares e drogas: comprovação de ação ergogênica e potenciais riscos para a saúde. Rev Bras Med Esporte. 2003; 8(2):43-56.

5. Sobal J , Marquart LF. Vitamin/mineral supplements use among athletes: a review of the literature. Int J Sport Nutr. 1994;4:320-4.

6. Rosen O, Sudgat-Borgen J , Maehlum S. Supplement use and nutritional habits in Norwegian elite athletes. Scand J Med Sci Sports. 1999;9:28-35.
7. Burke LM, Read RSD. Dietary supplements in sport. Sports Med. 1993;15:43-65.

8. Burke LM. Positive drug test from supplements. Sports Sci. 2000;4(3):1-5.

9. WADA-AMA. Independent observers report Olympic Summer Games 2000. Montreal: WADA, 2001.

10. Corrigan B, Kazlauskas R. Medication use in athletes selected for doping control at the Sydney Olympics (2000). Clin J Sport Med. 2003;13(1): 33-40.

11. Geyer H, Parr MK, Mareck-Engelke U, Schrader Y, Schänzer W. Analysis of nonhormonal nutritional supplements for anabolic-androgenic steroids - Results of an international study. IntJ Sports Med. 2004;25:124-9.

12. Geyer H, Henze MK, Mareck-Engelke U, Wagner A, Schänzer W. Positive dopingfälle mit Norandrosteron durch verunreinigte Nahrungsergänzungsmittel. Dtsch ZSportmed. 2000;51,11:378-82.

13. D'Angelo C. WADA education symposium. Montevideo: WADA, 2005 